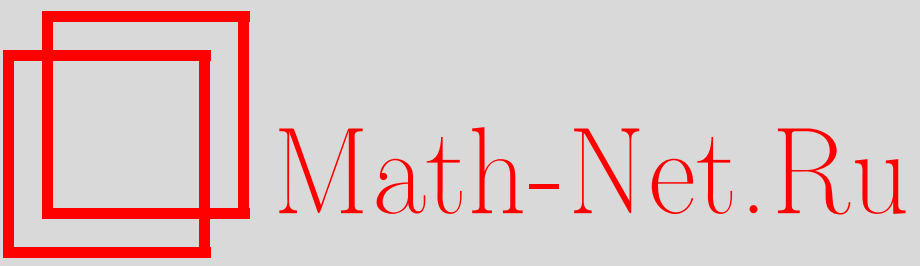

Э. Абадоглу, М. Бруски, Ф. Калоджеро, Азбука динамики магнитных монополей, ТМФ, 2001, том 128, номер 1, 4-14

DOI: https://doi.org/10.4213/tmf478

Использование Общероссийского математического портала Math-Net.Ru подразумевает, что вы прочитали и согласны с пользовательским соглашением

http://www.mathnet.ru/rus/agreement

Параметры загрузки:

IP: 54.172 .240 .79

26 апреля 2023 г., 17:17:20 
ТЕОРЕТИЧЕСКАЯ

И МАТЕМАТИЧЕСКАЯ

ФИЗИКА

Том 128, № 1

июль, 2001

(c) 2001 г. $\quad$ Е. Абадоглу* ${ }^{*}$ М. Бруски ${ }^{\dagger}$, Ф. Калоджеро ${ }^{\dagger}$

\section{АЗБУКА ДИНАМИКИ МАГНИТНЫХ МОНОПОЛЕЙ}

\footnotetext{
Приводится явное решение ньютоновских уравнений движения (сила равна массе, умноженной на ускорение) для точечного массивного магнитного монополя, движущегося в кулоновском поле неподвижного точечного электрического заряда, или, что эквивалентно, уравнений движения точечной массивной заряженной частицы, движущейся в магнитном поле неподвижного магнитного монополя.
}

\section{1. ВВЕДЕНИЕ}

Движение точечного магнитного монополя в кулоновском поле неподвижного точечного электрического заряда описывается уравнениями движения Ньютона-Лоренца

$$
\ddot{\vec{r}}=k r^{-3} \vec{r} \wedge \dot{\vec{r}}
$$

где $\vec{r} \equiv \vec{r}(t)$ - трехмерный вектор, описываюший положение движушейся частишы в момент времени $t$, точки над величинами обозначают дифференцирование по времени, символ $\wedge$ означает стандартное векторное произведение в трехмерном пространстве, a $r^{-3} \vec{r}$ - кулоновское поле неподвижного “единичного" электрического заряда, расположенного в начале координат. Тем самым правая часть уравнения (1.1) представляет собой силу Лоренца, действуюшую на движущийся монополь. Константа $k$, включающая в себя величины электрического и магнитного зарядов и массы движушейся частицы, имеет размерность (длина $)^{2}$ (время) $)^{-1}$ и может быть положена единицей при подходящем выборе системы отсчета (см. ниже). Точно такое же уравнение описывает движение точечной электрически заряженной частицы в магнитном поле неподвижного точечного монополя: поскольку до сих пор для монополей не нашлось места в списке реально обнаруженных элементарных частиц, эта вторая постановка задачи представляется несколько более реалистичной, ибо она, по крайней мере, описывает движение заряженной частицы в окрестности одного из двух полюсов магнита (вдали от второго полюса). Именно такая экспериментальная постановка задачи показалась интересной Пуанкаре, и он предложил полное и красивое решение этой задачи более ста лет

*Feza Gursey Institute, Kandilli, Istanbul, Turkey. E-mail: abadoglu@hotmail.com

${ }^{\dagger}$ Dipartimento di Fisica, Università di Roma "La Sapienza," Roma, Italy; Istituto Nazionale di Fisica Nucleare, Sezione di Roma. E-mail: bruschi@roma1.infn.it, francesco.calogero@roma1.infn.it 
назад [1]. K сожалению, это решение не так широко известно, как оно того заслуживает будучи единственным известным примером настояшего трехмерного движения (т.е. не в одной плоскости), которое может быть описано в терминах әлементарных функций; напомним, что њютоновское движение в поле центральных сил всегда происходит в плоскости. Однако поскольку сила Лоренца поперечна направлению движения, плоское движение не характерно для решений уравнения (1.1) (см. ниже). Более того, среди всех задач о ньютоновском движении (точечного массивного) монополя в центральном электрическом поле только случай кулоновского потенциала (см. (1.1)) допускает явное решение через элементарные функции. Ниже будет показано, что это свойство связано с замечательным фактом сушествования нетривиального неавтономного линейного обыкновенного дифференциального уравнения (ОДУ) третьего порядка, все решения которого выражаются в терминах элементарных функций [2], [3].

Проблема, которая вновь поднимается и исследуется в данной работе, уже несколько раз исследовалась в литературе (хотя указание на ее связь с явно решаемым ОДУ третьего порядка представляется новым); направления исследований в основном связывались с различными обобшениями данной задачи на случай, когда движушаяся частища и/или неподвижный источник поля несут как электрический, так и магнитный заряды (и тогда могут возникать дополнительные силы - см., например, недавнюю работу Сивардьере [4] и приведенную там литературу, а также работу Швингера и др. [5] и статью Гольдхабера и Трувера [6]). В настояшей работе ограничимся простейшим случаем, при котором движушаяся частица имеет магнитный заряд, а неподвижный источник силы генерирует электрическое поле (или наоборот), но сначала в разделе 2 исследуем случай центрального (не обязательно кулоновского) поля, а кулоновский случай будет рассмотрен отдельно в разделе 3 .

\section{2. ДВИЖЕНИЕ МАГНИТНОГО МОНОПОЛЯ В ЦЕНТРАЛЬНОМ ЭЛЕКТРИЧЕСКОМ ПОЛЕ}

Движение магнитного монополя в центральном электрическом поле описывается уравнением Ньютона-Лоренца

$$
\ddot{\vec{r}}=g(r) \vec{r} \wedge \dot{\vec{r}},
$$

которое преврашается в уравнение (1.1) в кулоновском случае, когда $g(r)=k r^{-3}$. Очевидно, это уравнение имеет решение

$$
\vec{r}(t)=\vec{r}(0)[1+\alpha t]
$$

( $\alpha$ - произвольная константа), описываюшее частицу, движушуюся в точности по радиусу и потому движушуюся свободно, так как сила Лоренца не действует в этом случае. В дальнейшем этот случай тривиального движения исключается из рассмотрения.

Из уравнения (2.1) немедленно следует закон сохранения

$$
\dot{\vec{r}} \cdot \ddot{\vec{r}}=0,
$$

откуда получаем, что квадрат скорости $\dot{\vec{r}} \cdot \dot{\vec{r}}=|\dot{\vec{r}}|^{2}$ является интегралом движения

$$
|\dot{\vec{r}}|=v \text {. }
$$


Очевидный физический смысл этого закона сохранения состоит в том, что сила Лоренца действует в направлении, поперечном направлению движения.

Кроме того, из уравнения (2.1) получим

$$
\vec{r} \cdot \ddot{\vec{r}}=0,
$$

и потому

$$
\frac{d^{2}\left[r^{2}(t)\right]}{d t^{2}}=\frac{2 d[\vec{r}(t) \cdot \dot{\vec{r}}(t)]}{d t}=2[\dot{\vec{r}}(t) \cdot \dot{\vec{r}}(t)]=2|\dot{\vec{r}}(t)|^{2}
$$

(см. (2.2)). Таким образом, после интегрирования получим формулу

$$
\vec{r}(t) \cdot \dot{\vec{r}}(t)=b+v^{2} t
$$

Поскольку нетрудно заметить, что

$$
\frac{d\left[r^{2}(t)\right]}{d t}=2 \vec{r}(t) \cdot \dot{\vec{r}}(t)
$$

то используя (2.3) и повторно проинтегрировав, получим

$$
r^{2}(t)=a^{2}+2 b t+v^{2} t^{2}
$$

где

$$
\begin{gathered}
a=r(0), \\
b=r(0) \dot{r}(0)=\vec{r}(0) \cdot \dot{\vec{r}}(0) .
\end{gathered}
$$

Качественный характер движения очевидно следует из (2.4): движущаяся частица приходит из бесконечности, достигает минимального расстояния от начала координат и затем уходит снова в бесконечность; вдали от источника частица движется почти по радиусу и соответственно свободно, поскольку сила Лоренца становится при этом мала из-за квазипараллельности $\vec{r}(t)$ и $\dot{\vec{r}}(t)$. Заметим, что это наблюдение не зависит от формы потенциала (центрального) электрического поля, которое даже может расти на бесконечности.

За начало отсчета по времени $(t=0)$ удобно взять момент времени $t_{c}=-b / v^{2}$ (см. (2.4)), когда движушаяся частица находится на минимальном расстоянии от центра координат. При этом

$$
b=0,
$$

а уравнение (2.4) принимает вид

$$
r(t)=\left(a+v^{2} t^{2}\right)^{\frac{1}{2}}
$$

В этом уравнении константа $a$ имеет физический смысл "минимального расстояния". Таким образом, обе константы в выражении (2.8) для временно́й зависимости расстояния движушейся частищы от источника поля имеют явный физический смысл. 
Из формул (2.2), (2.3) и (2.4) следует, что

$$
(\vec{r} \cdot \vec{r})(\dot{\vec{r}} \cdot \dot{\vec{r}})-(\vec{r} \cdot \dot{\vec{r}})^{2}=a^{2} v^{2}-b^{2}
$$

(независимо от (2.7)!). Величина в левой части этого уравнения является вторым интегралом движения (в дополнение к (2.2)). Физический смысл этого интеграла - это квадрат модуля углового момента движушейся частицы,

$$
\vec{L}=\vec{r} \wedge \dot{\vec{r}}
$$

поскольку из этого определения следует, что

$$
L^{2}=(\vec{r} \cdot \vec{r})(\dot{\vec{r}} \cdot \dot{\vec{r}})-(\vec{r} \cdot \dot{\vec{r}})^{2}
$$

При условии (2.7) получим

$$
L^{2}=a^{2} v^{2}
$$

и это равенство также допускает очевидную физическую интерпретацию. В момент времени, когда движушаяся частиша достигает точки, максимально приближенной к началу координат (момент времени $t=0$ ), ее вектор скорости ортогонален ее радиусвектору (см. (2.6) и (2.7)), и тем самым модуль углового момента (второй интеграл движения) равен простому произведению наименьшего расстояния $a$ и скорости $v$ (также являюшейся постоянной величиной).

Независимость $L^{2}$ от времени может быть доказана непосредственно. Для этого продифференцируем уравнение (2.9) по времени и получим

$$
\dot{\vec{L}}=\vec{r} \wedge \ddot{\vec{r}}
$$

а потому с помошью уравнения (2.1) получим, что

$$
\dot{\vec{L}}=g(r)\left[\vec{r}(\vec{r} \cdot \dot{\vec{r}})-\dot{\vec{r}} r^{2}\right],
$$

где использовано известное тождество для трех векторов (в трехмерном пространстве)

$$
\vec{r}_{1} \wedge\left(\vec{r}_{2} \wedge \vec{r}_{3}\right)=\left(\vec{r}_{1} \cdot \vec{r}_{3}\right) \vec{r}_{2}-\left(\vec{r}_{1} \cdot \vec{r}_{2}\right) \vec{r}_{3}
$$

Тогда из соотношения (2.9) (которое отражает тот факт, что вектор $\vec{L}$ ортогонален как $\vec{r}$, так и $\dot{\vec{r}})$ следует формула

$$
\vec{L} \cdot \dot{\vec{L}}=0
$$

которая явно показывает, что производная по времени от $L^{2}(t)$ равна нулю и, следовательно, $L^{2}$ является интегралом движения.

До сих пор исследовались лишь несколько простых свойств движения, задаваемого уравнением (2.1), которое однако не столь уж и просто ввиду своей явной трехмерности, обусловленной поперечностью силы Лоренца. Тем не менее имеет место еще одно замечательное упрошение, которое возникает, если продифференцировать уравнение (2.1) по времени. После этого получим

$$
\dddot{\vec{r}}=\frac{g^{\prime}(r)}{g(r)} \dot{\vec{r}}+g(r) \vec{r} \wedge \ddot{\vec{r}},
$$


и из уравнения (2.1) и тождества (2.12) следует, что

$$
\dddot{\vec{r}}=\frac{g^{\prime}(r)}{g(r)} \dot{\vec{r}} \ddot{\vec{r}}+[g(r)]^{2}\left[\vec{r}(\vec{r} \cdot \dot{\vec{r}})-\dot{\vec{r}} r^{2}\right]
$$

откуда, подставив $(2.8)$ с учетом $(2.3)$ и $(2.7)$, имеем

$$
\dddot{\vec{r}}=f(t) \ddot{\vec{r}}+h(t)\left[\vec{r} v^{2} t-\dot{\vec{r}} r^{2}\right],
$$

где

$$
\begin{aligned}
& f(t)=\frac{v^{2} t\left(a^{2}+v^{2} t^{2}\right)^{-\frac{1}{2}} g^{\prime}\left[\left(a^{2}+v^{2} t^{2}\right)^{\frac{1}{2}}\right]}{g\left[\left(a^{2}+v^{2} t^{2}\right)^{\frac{1}{2}}\right]}, \\
& h(t)=g^{2}\left[\left(a^{2}+v^{2} t^{2}\right)^{\frac{1}{2}}\right] .
\end{aligned}
$$

Из уравнения (2.14) следует, что каждая из трех компонент радиус-вектора $\vec{r}(t)$ или, что эквивалентно, каждая величина (например, $\chi(t)$ ), пропорциональная какой-либо компоненте вектора $\vec{r}(t)$, удовлетворяет линейному неавтономному ОДУ третьего порядка, которое может быть представлено в удобном виде как

$$
\chi^{\prime \prime \prime}(\tau)+\alpha(\tau) \chi^{\prime \prime}(\tau)+\beta(\tau)\left[\left(1+\tau^{2}\right) \chi^{\prime}(\tau)-\tau \chi(\tau)\right]=0,
$$

где введены обозначения

$$
\begin{aligned}
\tau & =t \frac{v}{a} \\
\alpha(\tau) & =-\frac{a}{v} f(t), \quad \beta(\tau)=\frac{a^{4}}{v^{2}} h(t) .
\end{aligned}
$$

Здесь и ниже штрихи обозначают дифференцирование по переменной $\tau$.

В частности, если $g(r)($ см. (2.1)) имеет степенную зависимость,

$$
g(r)=k r^{p}
$$

то (см. $(2.15)$ и $(2.18))$

$$
\alpha(\tau)=-p \frac{\tau}{1+\tau^{2}}, \quad \beta(\tau)=\lambda\left(1+\tau^{2}\right)^{p}
$$

где

$$
\lambda=k \frac{a^{p+2}}{v} .
$$

Заметим, что масштабированные время $\tau$ (см. (2.17)) и константа связи $\lambda$ становятся безразмерными.

Теперь можно фиксировать произвол в трех независимых решениях $\chi_{0}(\tau), \chi_{1}(\tau)$ и $\chi_{2}(\tau)$ ОДУ третьего порядка (2.16) с помошью задания начальных условий

$$
\begin{array}{lll}
\chi_{0}(0)=1, & \chi_{0}^{\prime}(0)=0, & \chi_{0}^{\prime \prime}(0)=0, \\
\chi_{1}(0)=0, & \chi_{1}^{\prime}(0)=1, & \chi_{1}^{\prime \prime}(0)=0, \\
\chi_{2}(0)=0, & \chi_{2}^{\prime}(0)=0, & \chi_{2}^{\prime \prime}(0)=1 .
\end{array}
$$


Если известны три указанных решения, то решение задачи (2.1) с заданными начальными данными дается явной формулой

$$
\vec{r}(t)=\chi_{0}(\tau) \vec{r}(0)+\frac{a}{v} \chi_{1}(\tau) \dot{\vec{r}}(0)+\left(\frac{a}{v}\right)^{2} \chi_{2}(\tau) g[r(0)] \vec{r}(0) \wedge \dot{\vec{r}}(0),
$$

что легко проверить с использованием формул (2.1), (2.17) и (2.21).

В разделе 3 будет показано, что в специальном случае $p=-3$, который отвечает ввиду (2.19) и (2.1) "физическому” случаю реального кулоновского потенциала (см. (1.1)), линейное ОДУ третьего порядка (2.16) может быть явно решено в терминах элементарных функций. Но прежде отметим еше один замечательный факт, имеюший место для произвольной функции $g(r)$ (см. (2.1)). Рассмотрим скалярную величину

$$
\Delta(t)=[\vec{r}(t) \wedge \dot{\vec{r}}(t)] \cdot \ddot{\vec{r}}(t),
$$

которая имеет ясный геометрический смысл: с точностью до множителя $1 / 6$ и, возможно, знака эта величина есть объем тетраэдра, ребрами которого являются векторы $\vec{r}(t)$, $\dot{\vec{r}}(t)$ и $\ddot{\vec{r}}(t)$. Из определения $(2.23)$ явно следует

$$
\dot{\Delta}(t)=[\vec{r}(t) \wedge \dot{\vec{r}}(t)] \cdot \dddot{\vec{r}}(t),
$$

а потому из (2.13) и (2.23) получим

$$
\dot{\Delta}(t)=\frac{g^{\prime}[r(t)]}{g[r(t)]} \dot{r}(t) \Delta(t) .
$$

Поделив это выражение на $\Delta(t)$ с последуюшим интегрированием, получим следуюшую формулу:

$$
\Delta(t)=c g[r(t)],
$$

где константа задается выражением

$$
c=\frac{\Delta(0)}{g[r(0)]} .
$$

С другой стороны, из определения (2.23) для величины $\Delta(t)$ и формул $(2.1)$ и $(2.9)$ следует, что

$$
\Delta(t)=g[r(t)]|\vec{L}(t)|^{2} .
$$

Сравнивая эту формулу с (2.24), можно опять заключить, что абсолютная величина углового момента $\vec{L}$ (см. (2.9)) есть интеграл движения,

$$
|\vec{L}(t)|^{2}=|\vec{L}(0)|^{2}=(a v)^{2} .
$$

\section{3. РЕШЕНИЕ В КУЛОНОВСКОМ СЛУЧАЕ}

Исследуем движение точечного (массивного) монополя в кулоновском электрическом поле неподвижного электрического заряда (расположенного в начале координат) или, что эквивалентно, движение точечной (массивной) электрически заряженной 
частицы в магнитном поле неподвижного магнитного монополя. Уравнение НьютонаЛоренца указанной системы имеет вид (1.1), где $k$ - это константа (пропорциональная электрическому и магнитному зарядам и обратно пропорциональная массе движушейся частицы), имеющая размерность (длина $)^{2}$ (время) ${ }^{-1}$.

Как показано в разделе 2, уравнения (2.1) и, следовательно, (1.1) приводят к двум законам сохранения

$$
\begin{gathered}
|\dot{\vec{r}}(t)|=|\dot{\vec{r}}(0)|=v, \\
r^{2}(t)=a^{2}+2 b t+v^{2} t^{2},
\end{gathered}
$$

где константы $v, a^{2}$ и $b$ определяются формулами (3.1), (2.5) и (2.6). Как и в разделе 2 , будем предполагать (с существенным упрошением обозначений и без потери обшности), что в момент времени $t=0$ частица находится на минимальном расстоянии от центра сил; в этот же момент времени вектор скорости ортогонален радиус-вектору. Тем самым $b=0($ см. $(2.7))$, и вместо (3.2) получим

$$
r^{2}(t)=a^{2}+v^{2} t^{2}
$$

Преимушество этого представления состоит в том, что обе константы в нем имеют ясный физический смысл: $v$ - это постоянная величина модуля скорости движущейся вдоль своей траектории частицы, а $a$ - минимальное расстояние от этой траектории до начала координат. (Как и в разделе 2, тривиальное радиальное свободное движение исключается, т.е. по предположению $a>0$. Также исключен тривиальный случай неподвижной частицы, т.е. предполагается, что $v>0$.)

Развивая эти (вполне обшие) предположения, удобно выбрать расстояние $a$ в качестве масштаба расстояния, а величину (см. (2.17))

$$
T=\frac{a}{v}
$$

имеющую размерность времени, положить единицей его измерения. Таким образом, можно ввести безразмерные величины $\tau, \vec{\rho}$ и $\lambda$ :

$$
t=T \tau, \quad \vec{r}(t)=a \vec{\rho}(\tau), \quad k=\frac{a^{2}}{T} \lambda=a v \lambda
$$

(ср. (2.17) и (2.20)). Эти величины выбраны таким образом, чтобы совместно анализировать семейства траекторий частиц, имеющих одинаковые скорость $v$ и минимальное расстояние $a$ до начала координат. В дальнейшем точки над величинами будут обозначать дифференцирование по безразмерному времени $\tau$. Уравнения движения (1.1) теперь примут вид

$$
\ddot{\vec{\rho}}=\lambda \rho^{-3} \vec{\rho} \wedge \dot{\vec{\rho}},
$$

в то время как (см. (3.1) и (3.4))

$$
|\dot{\vec{\rho}}(\tau)|=1
$$

и (см. (3.3))

$$
\rho^{2}(\tau)=1+\tau^{2} .
$$


Удобно также ввести “безразмерный угловой момент” $\vec{\Lambda}$ движущейся частицы:

$$
\vec{\Lambda}=\frac{\vec{L}}{a v}=\vec{\rho} \wedge \dot{\vec{\rho}}
$$

(см. (2.11), (3.4) и (3.5)). Заметим, что согласно результатам раздела 2 абсолютная величина этого безразмерного вектора есть константа, равная единице в выбранной системе единиц,

$$
\Lambda^{2}=1
$$

(см. $(2.10)$ и (3.9)).

С другой стороны, сам угловой момент не сохраняется в отличие от случая частищы, движушейся в поле центральных сил. (Сила Лоренца в правой части (3.6), равно как и в (2.1), не является центральной.) Однако в случае кулоновских сил, рассматриваемом в этом разделе, существует сохраняющийся трехмерный вектор

$$
\begin{aligned}
\vec{J} & =\vec{\Lambda}+\lambda \hat{\rho}, \\
\dot{\vec{J}} & =0 .
\end{aligned}
$$

Здесь и ниже

$$
\hat{\rho} \equiv \frac{\vec{\rho}}{\rho}
$$

есть единичный вектор в направлении $\vec{\rho}$. Разумеется, вектор $\vec{J}$, равно как и $\vec{\Lambda}, \lambda$ и $\hat{\rho}$, безразмерен.

Приведем доказательство формулы (3.11б):

$$
\begin{aligned}
& \dot{\vec{J}}=\dot{\vec{\Lambda}}+\lambda\left[\rho^{-1} \dot{\vec{\rho}}-\rho^{-2} \dot{\rho} \vec{\rho}\right], \\
& \dot{\vec{\Lambda}}=\vec{\rho} \wedge \ddot{\vec{\rho}} \\
& \dot{\vec{\Lambda}}=\lambda \rho^{-3} \vec{\rho} \wedge(\vec{\rho} \wedge \dot{\vec{\rho}}), \\
& \dot{\vec{\Lambda}}=-\lambda \rho^{-1} \dot{\vec{\rho}}+\lambda \rho^{-3}(\vec{\rho} \cdot \dot{\vec{\rho}}) \vec{\rho}, \\
& \dot{\vec{\Lambda}}=-\lambda\left[\rho^{-1} \dot{\vec{\rho}}-\rho^{-2} \dot{\rho} \vec{\rho}\right],
\end{aligned}
$$

где (3.14а) следует из (3.9) после дифференцирования по $\tau$; формулу (3.14б) можно получить из (3.14a) с помощью уравнений движения $(3.6) ;(3.14 \mathrm{~B})$ получается из $(3.14 \sigma)$ с учетом векторного тождества $(2.12) ;(3.14$ г) следует из $(3.14$ в) и соотношения $\rho \dot{\rho}=\vec{\rho} \cdot \dot{\vec{\rho}}$ (которое, естественно, может быть получено в результате дифференцирования величины $\rho^{2}=\vec{\rho} \cdot \vec{\rho}$ по времени). Тогда из формул $(3.14 \Gamma)$ и (3.13) следует искомое соотношение $(3.116)$.

То, что вектор $\vec{J}$ есть сохраняющаяся величина для уравнения (3.6), было показано еше Пуанкаре более ста лет назад [1]; вектор $\vec{J}$ называется вектором Пуанкаре.

Поскольку векторы $\vec{\Lambda}$ и $\hat{\rho}$ ортогональны (см. (3.9) и (3.12)), из определения (3.11a) вектора Пуанкаре следует, что

$$
J^{2}=\Lambda^{2}+\lambda^{2},
$$


равно как и

$$
\hat{\rho} \cdot \vec{J}=\lambda .
$$

Угол $\theta$ между единичным вектором $\hat{\rho} \equiv \hat{\rho}(\tau)$ (или, что эквивалентно, трехмерным вектором $\vec{\rho} \equiv \vec{\rho}(\tau))$ и постоянным вектором $\vec{J}$ также сохраняется и дается равенством

$$
\cos \theta=\frac{\lambda}{J}
$$

Таким образом, движение происходит на фиксированном круговом полуконусе с (постоянным) половинным углом $\theta$, заданным соотношением (3.16), с вершиной в начале координат и с осью, совпадающей с направлением (постоянного) трехмерного вектора $\vec{J}$. Тем самым, если выбрать $\rho \equiv \rho(\tau), \theta$ и $\varphi \equiv \varphi(\tau)$ в качестве сферических координат движущейся частищы в координатной системе, начало которой совпадает с положением неподвижной частищы, а азимутальная ось совпадает с направлением вектора $\vec{J}$, то получим

$$
\vec{\rho}(\tau) \equiv \rho(\tau)(\sin \theta \cos \varphi(\tau), \sin \theta \sin \varphi(\tau), \cos \theta) .
$$

Теперь осталось только найти $\varphi(\tau)$, чтобы получить полную информацию о движении частицы, т.е. о векторе $\vec{\rho}(\tau)$, потому что $\rho(\tau)$ задается уравнением $(3.8)$, а угол $\theta$ остается постоянным в процессе движения. Равенство (3.16) вместе с формулами (3.15) и (3.10) дает соотношение

$$
\cos \theta=\lambda\left(1+\lambda^{2}\right)^{-\frac{1}{2}}
$$

и, таким образом,

$$
\begin{aligned}
\sin \theta & =\left(1+\lambda^{2}\right)^{-\frac{1}{2}}, \\
\operatorname{ctg} \theta & =\lambda .
\end{aligned}
$$

Теперь можно легко вычислить $\varphi(\tau)$. Действительно, из соотношения (3.17) получим

$$
\dot{\vec{\rho}}=\dot{\rho}(\sin \theta \cos \varphi, \sin \theta \sin \varphi, \cos \theta)+\rho \dot{\varphi}(-\sin \theta \sin \varphi, \sin \theta \cos \varphi, 0) .
$$

Следовательно,

$$
\dot{\vec{\rho}} \cdot \dot{\vec{\rho}}=\left(\dot{\rho}^{2}+\rho^{2} \dot{\varphi}^{2}\right) \sin ^{2} \theta+\dot{\rho}^{2} \cos ^{2} \theta .
$$

С использованием (3.7) получим

$$
1=\dot{\rho}^{2}+\rho^{2} \dot{\varphi}^{2} \sin ^{2} \theta
$$

откуда с привлечением (3.8) и (3.18б) легко получить, что

$$
1=\frac{\tau^{2}}{1+\tau^{2}}+\dot{\varphi}^{2} \frac{1+\tau^{2}}{1+\lambda^{2}},
$$

что и дает соотношение

$$
\dot{\varphi}(\tau)=\frac{\left(1+\lambda^{2}\right)^{\frac{1}{2}}}{1+\tau^{2}} .
$$

Интегрируя (3.19), получим

$$
\varphi(\tau)=\varphi_{0}+\left(1+\lambda^{2}\right)^{\frac{1}{2}} \operatorname{arctg} \tau,
$$


где $\varphi_{0}=\varphi(0)$.

Если использовать выражение (3.17), то формулы (3.8), (3.18) и (3.20) дают полную информацию о движении частицы, которое описывается явно и в терминах элементарных функций.

Те же результаты могут быть получены, если заметить, что в случае $p=-3$ (когда уравнение (2.1) с функцией $g(r)(2.19)$ совпадает с (1.1)) линейное ОДУ третьего порядка (2.16) полностью решается в терминах элементарных функций. В этом случае это уравнение, записанное для каждой компоненты $\psi$ трехмерного вектора $\vec{\rho}$ в отдельности, имеет вид

$$
\left(1+\tau^{2}\right)^{3} \dddot{\psi}+3 \tau\left(1+\tau^{2}\right)^{2} \ddot{\psi}+\lambda^{2}\left(1+\tau^{2}\right) \dot{\psi}-\lambda^{2} \tau \psi=0
$$

Три независимых решения этого уравнения даются формулами

$$
\psi_{s}(\tau)=(1+i \tau)^{\frac{1+s \gamma}{2}}(1-i \tau)^{\frac{1-s \gamma}{2}}=\left(1+\tau^{2}\right)^{\frac{1}{2}} e^{i s \gamma \operatorname{arctg} \tau}, \quad s=0, \pm 1,
$$

где

$$
\gamma=\left(1+\lambda^{2}\right)^{\frac{1}{2}}
$$

Действительно, из (3.22) легко получить, что

$$
\begin{aligned}
& \frac{\dot{\psi}_{s}(\tau)}{\psi_{s}(\tau)}=(i s \gamma+\tau)\left(1+\tau^{2}\right)^{-1}, \\
& \frac{\ddot{\psi}_{s}(\tau)}{\psi_{s}(\tau)}=\left(1-s^{2} \gamma^{2}\right)\left(1+\tau^{2}\right)^{-2}, \\
& \frac{\dddot{\psi}_{s}(\tau)}{\psi_{s}(\tau)}=\left(1-s^{2} \gamma^{2}\right)(i s \gamma-3 \tau)\left(1+\tau^{2}\right)^{-3} .
\end{aligned}
$$

Подставив эти выражения в (3.21), получим

$$
s \gamma\left[s^{2} \gamma^{2}-\left(1+\lambda^{2}\right)\right]=0,
$$

и из формулы (3.23) следует, что

$$
s\left(s^{2}-1\right)=0 .
$$

Таким образом, анзац (3.22) удовлетворяет уравнению (3.21) при $s=0, s=+1$ и $s=$ -1 .

Внимательный читатель легко получит конечное решение поставленной задачи, используя выражения (2.21) и (2.22) и сравнивая их с вышеприведенными решениями (3.22).

В заключение представим окончательный результат для траекторий в терминах исходных переменных (начало отсчета по времени по-прежнему совпадает с моментом, когда частица максимально приближается к началу координат). Результаты легче всего представить (и проанализировать) в сферических координатах в системе отсчета, описанной выше, в которой начало координат совпадает с неподвижной частицей, а азиму- 
тальная ось $z$ параллельна вектору Пуанкаре $\vec{J}$ (см. ((3.11), (3.9) и (3.12)):

$$
\begin{aligned}
\vec{r}(t) & =r(t)(\sin \theta \cos \phi(t), \sin \theta \sin \phi(t), \cos \theta), \\
r(t) & =a\left[1+\left(\frac{t}{T}\right)^{2}\right]^{\frac{1}{2}} \\
\theta & =\operatorname{arcctg} \lambda \\
\phi(t) & =\phi_{0}+\left(1+\lambda^{2}\right)^{\frac{1}{2}} \operatorname{arctg} \frac{t}{T}
\end{aligned}
$$

где $T$ и $\lambda$ даются соответственно формулами (3.4) и (3.5). Из вышеприведенных формул следует, что в асимптотическом режиме (в отдаленных прошлом и будушем) все траектории становятся прямыми линиями, а именно при $t \rightarrow \pm \infty$ получим

$$
\vec{r}(t)=\vec{v}_{ \pm} t+\vec{r}_{ \pm}^{(0)}+O\left(t^{-1}\right) .
$$

Также легко видеть, что из этих формул следует

$$
\begin{gathered}
b_{ \pm}=r_{ \pm}^{(0)} \equiv\left|\vec{r}_{ \pm}^{(0)}\right|=a \\
\cos \alpha=\frac{\vec{v}_{+} \cdot \vec{v}_{-}}{v^{2}}=-1+2\left(1+\lambda^{2}\right)^{-1} \sin ^{2} \frac{\left(1+\lambda^{2}\right)^{\frac{1}{2}} \pi}{2} \\
\Delta \phi \equiv \phi(\infty)-\phi(-\infty)=\pi\left(1+\lambda^{2}\right)^{\frac{1}{2}}
\end{gathered}
$$

где, разумеется, асимптотические скорости имеют одинаковые абсолютные значения, $v_{ \pm} \equiv\left|\vec{v}_{ \pm}\right|=v$, величины $b_{ \pm}$(см. (3.25)) суть прицельные параметры (расстояния от начала координат до асимптот; см. (3.24)), величина $\alpha$, которая определяется по модулю $2 \pi$ первым равенством в формуле $(3.26)$, представляет собой угол рассеяния, а величина $\Delta \phi$ (см. (3.27)) задает полное врашение траектории в направлении угла $\phi$.

Благодарности. Авторы благодарны М. Атье, К. Бернардини, Н. Мантону и $\Phi$. Рерлиху за помощь в освещении истории исследуемой задачи, а также рецензенту и М. Деминову, заметившим нетривиальную ошибку в знаке в промежуточной версии данной работы.

\section{Список литературы}

[1] H. Poincaré. Compt. Rendus Acad. Sci. 1986. V. 123. P. 530-533.

[2] M. Bruschi, F. Calogero. J. Nonlinear Math. Phys. 2000. V. 7. P. 303-386.

[3] F. Calogero. Classical Many-Body Problems Amenable to Exact Treatments. Lect. Notes Phys. Monograph. V. 66. Berlin: Springer, 2001.

[4] J. Sivardière. Eur. J. Phys. 2000. V. 21. P. 183-190.

[5] J. Schwinger, K. A. Milton, Wu-yang Tsai, L. L. DeRaad Jr., D. C. Clark. Ann. Phys. 1976. V. 101. P. 451-495.

[6] A. S. Goldhaber, W. P. Trower. Am. J. Phys. 1990. V. 58. P. 429-439. 